\title{
PREDICTION OF THE SPECTROSCOPIC PARAMETERS OF NEW IRON COMPOUNDS: HYDRIDE OF IRON CYANIDE/ISOCYANIDE, HFeCN/HFeNC
}

\author{
Pilar Redondo, Carmen Barrientos, and Antonio Largo \\ Departamento de Química Física y Química Inorgánica Facultad de Ciencias, Universidad de Valladolid Campus Miguel Delibes Paseo de Belén 7, E-47011, \\ Valladolid, Spain; predondo@qf.uva.es \\ Received 2016 January 14; revised 2016 May 3; accepted 2016 July 11; published 2016 August 26
}

\begin{abstract}
Iron is the most abundant transition metal in space. Its abundance is similar to that of magnesium, and until today only, $\mathrm{FeO}$ and $\mathrm{FeCN}$ have been detected. However, magnesium-bearing compounds such as $\mathrm{MgCN}, \mathrm{MgNC}$, and $\mathrm{HMgNC}$ are found in IRC +10216 . It seems that the hydrides of iron cyanide/isocyanide could be good candidates to be present in space. In the present work we carried out a characterization of the different minima on the quintet and triplet $[\mathrm{C}, \mathrm{Fe}, \mathrm{H}, \mathrm{N}]$ potential energy surfaces, employing several theoretical approaches. The most stable isomers are predicted to be hydride of iron cyanide $\mathrm{HFeCN}$, and isocyanide $\mathrm{HFeNC}$, in their ${ }^{5} \Delta$ states. Both isomers are found to be quasi-isoenergetics. The HFeNC isomer is predicted to lie about $0.5 \mathrm{kcal} / \mathrm{mol}$ below $\mathrm{HFeCN}$. The barrier for the interconversion process is estimated to be around $6.0 \mathrm{kcal} / \mathrm{mol}$, making this process unfeasible under low temperature conditions, such as those in the interstellar medium. Therefore, both $\mathrm{HFeCN}$ and $\mathrm{HFeNC}$ could be candidates for their detection. We report geometrical parameters, vibrational frequencies, and rotational constants that could help with their experimental characterization.
\end{abstract}

Key words: astrochemistry - ISM: general - ISM: molecules - ISM: structure - molecular data

\section{INTRODUCTION}

Knowledge of metal-containing molecule chemistry in the interstellar medium has evolved as more and more systems have been detected. Initially, it was expected that metal halides species of elements such as $\mathrm{Na}, \mathrm{K}, \mathrm{Mg}, \mathrm{Al}$, and Fe were present only in the hot photospheres of stars, while in circumstellar envelopes of evolved stars species were formed with $\mathrm{C}, \mathrm{O}, \mathrm{N}$, and Si atoms (Tsuji 1973). However, in 1987 the metal halide compounds $\mathrm{NaCl}, \mathrm{AlCl}, \mathrm{KCl}$, and $\mathrm{AlF}$ were detected in the circumstellar envelope of carbon-rich star IRC +10216 by Cernicharo \& Guélin (1987). Subsequently, the metal cyanide compounds MgNC (Guélin et al. 1993; Kawaguchi et al. 1993), $\mathrm{NaCN}$ (Turner et al. 1994), MgCN (Ziurys et al. 1995), SiCN (Guélin et al. 2000), AlNC (Ziurys et al. 2002), SiNC (Guélin et al. 2004), KCN (Pulliam et al. 2010), and FeCN (Zack et al. 2011), were detected in IRC +10216 . Related to these compounds, recently the first hydro-metal cyanide, $\mathrm{HMgCN}$, was detected in this source (Cabezas et al. 2013). Other metaloxygen-containing compounds such as $\mathrm{FeO}, \mathrm{AlO}, \mathrm{AlOH}, \mathrm{TiO}$, and $\mathrm{TiO}_{2}$, were also detected in different sources (Walmsley et al. 2002; Tenenbaum \& Ziurys 2009, 2010; Kamiński et al. 2013).

Nowadays, taking into account the characterized species, the chemistry of metal-containing molecules in carbon-rich circumstellar gas, especially in IRC +10216 , seems to be controlled by cyanides/isocyanides. This dominant role was not established by any chemical model and it can evolve as more molecules are detected. Recently, Hernández Vera et al. (2015; see also the references therein) studied cyanide/ isocyanide abundances in terms of molecular stabilities, reaction probabilities, and radiative and collisional excitations. Future detection of metal compounds will provide information that could help us to understand where and how the different species are formed. It is also interesting to take into account the fact that cyanogen $(\mathrm{CN})$ is the simplest molecule including carbon and nitrogen, essential elements in the chemistry of life. Metal-CN bonds are common in nature as examples, Vitamin
B12 contains the Co-CN moiety, and the well-known dye "Prussian Blue," whose molecular formula can be written as $\left[\mathrm{Fe}_{4}\left[\mathrm{Fe}(\mathrm{CN})_{6}\right]_{3}\right]$, is formed from $\mathrm{FeCN}$ units.

Hydromagnesium isocyanide, $\mathrm{HMgNC}$, is the only hydride of a metal cyanide/isocyanide detected in space (Cabezas et al. 2013). Theoretical results and laboratory characterization have been reported for $\mathrm{HSiCN} / \mathrm{HSiNC}$ and $\mathrm{HZnCN} / \mathrm{HZnNC}$ systems. In the case of the hydrides of silicon, the rotational spectra of both isomers are characterized in the laboratory (Sanz et al. 2002), and an ab initio calculation predicts that both isomers are close in energy; the isocyano isomer HSiNC is located only $3.2 \mathrm{kcal} / \mathrm{mol}$ below the $\mathrm{HSiCN}$ isomer (Maier et al. 1998). For zinc, only the structure of the hydride of zinc cyanide, $\mathrm{HZnCN}$, is characterized using Fourier transform microwave spectroscopy (Sun et al. 2009). A recent theoretical study of $[\mathrm{C}, \mathrm{H}, \mathrm{N}, \mathrm{Zn}]$ isomers predicts that $\mathrm{HZnCN}$ is the lowest-lying isomer and the isocyanide counterpart $\mathrm{HZnNC}$ is only located $5.13 \mathrm{kcal} / \mathrm{mol}$ (at $\operatorname{CCSD}(\mathrm{T}) /$ aug-cc-pVQZ// CCSD/aug-cc-pVTZ level) above $\mathrm{HZnCN}$ (Redondo et al. 2015).

Iron is the most abundant transition metal in space, with $\mathrm{Fe} /$ $\mathrm{H} \approx 310^{-5}$; however, until today only two molecules containing this metal have been detected, $\mathrm{FeO}$ and $\mathrm{FeCN}$. Its abundance is comparable to that of magnesium, of which $\mathrm{MgCN}, \mathrm{MgNC}$, and $\mathrm{HMgNC}$ are found in IRC +10216 . It seems that hydrides of iron cyanide/isocyanide could be good candidates to be present in space. In addition, Cabezas et al. (2013) argue that the presence of $\mathrm{MgCN}, \mathrm{MgNC}$ and $\mathrm{HMgNC}$ in the same source suggests a common origin. Based on the theoretical results of Petrie (1996) and Dunbar \& Petrie (2002) they consider that these compounds are formed in the gas phase by radiative association of $\mathrm{Mg}^{+}$and cyanopolyynes followed by the dissociative recombination of the cation complex.

The characterization of the ground state for iron compounds is a relatively difficult task because they usually have many states of different multiplicities with similar energy. Prior to the detection of $\mathrm{FeCN}$ in IRC +10216 there have been some 
discussions about the ground state and relative stability of the isomers $\mathrm{FeCN} / \mathrm{FeNC}$. The experimental study of Flory \& Ziurys (2011) established FeCN as the most stable species with a quartet ground state, ${ }^{4} \Delta$, while previous theoretical studies, such as those by DeYonker et al. (2004), Hirano et al. (2006), and Rayon et al. (2007), found that the sextet state of FeNC, ${ }^{6} \Delta$, is more stable. In a subsequent work Hirano et al. (2007) predicted the ${ }^{6} \Delta$ state of $\mathrm{FeCN}$ as the most stable isomer. Recently, high-level theoretical calculations (in particular coupled-cluster theory with post $\operatorname{CCSD}(\mathrm{T})$ additive corrections) by DeYonker (2015) predicted the ${ }^{4} \Delta$ state of FeCN as the ground state, according to the experimental results.

To the best of our knowledge there are no results, neither theoretical nor experimental, for hydrides of iron cyanide/ isocyanide, $\mathrm{HFeCN} / \mathrm{HFeNC}$. In the present work we provide a computational study of possible tetratomic $[\mathrm{C}, \mathrm{Fe}, \mathrm{H}, \mathrm{N}]$ isomers, making use of an accurate quantum chemical approach. We analyze the thermodynamic stability of the different isomers and for the most stables ones, HFeCN/ $\mathrm{HFeNC}$, we perform high-level calculations in order to provide information about their molecular structure and spectroscopic parameters. These predictions could be helpful for their eventual observation in the laboratory or in the interstellar medium. In addition, interconversion processes between isomers could provide some insight into the origin of these systems in space.

\section{COMPUTATIONAL METHODS}

The characterization of the possible $[\mathrm{C}, \mathrm{Fe}, \mathrm{H}, \mathrm{N}]$ isomers was carried out by employing different theoretical approaches. The geometries were optimized as a first approach at the density functional theory (DFT) level, employing the B3LYP functional (Becke 1988; Lee et al. 1988). For the most relevant structures characterized at the B3LYP level, we apply different ab initio methods, particularly second-order Møller-Plesset (MP2; Møller \& Plesset 1934), coupled-cluster with single and double excitations (CCSD), and CCSD(T), which also includes a perturbative treatment of triple excitations (Raghavachari et al. 1989).

On the optimized geometries harmonic vibrational frequencies were also calculated at their respective levels of theory, except for the CCSD(T) level. This allows us to estimate the zero-point vibrational energy correction (ZPE) and confirm the nature of the stationary points located on the potential energy surface, either a true minimum (all vibrational frequencies real) or a transition state (one of the frequencies, and just one, imaginary). We have not been able to calculate anharmonic corrections for vibrational frequencies. During the variational procedure (VPT2), symmetry is lowered from $\mathrm{C}_{\infty \mathrm{v}}$ to $\mathrm{C}_{\mathrm{s}}$ and the ground state collapses into other states. A similar behavior was found by DeYonker (2015) in the FeCN/FeNC systems.

Due to the large number of states that are close in energy that are present in this type of system, in order to establish the lowest-lying state for each multiplicity, a stability test was performed. In the CCSD(T) calculations, we estimated the T1 diagnostic (Lee \& Taylor 1989) to analyze the viability of single-reference results. In addition, a CASSCF (complete active space multiconfiguration self-consistent field; Werner \& Knowles 1985a, 1985b) calculation was performed for the two lowest-lying isomers, $\mathrm{HFeCN} / \mathrm{HFeNC}$.

Regarding basis sets we have used the Dunning's aug-ccpVTZ (correlation-consistent polarized valence triple-zeta including diffuse functions) basis set (Dunning 1989; Woon \& Dunning 1993) for optimization and harmonic frequencies calculations. Single-point energy calculations were also performed at the CCSD(T) level employing the aug-cc-pVQZ basis set (Dunning 1989; Woon \& Dunning 1993), which has a quadruple-zeta character and includes both diffuse and polarization functions.

As mentioned in the introduction, the characterization of the ground state for iron compounds is a relatively difficult task. In order to provide a more reliable description of the most stable isomers, additional calculations were performed for them. This way, the effect of core-valence correlation was accounted for by including $3 s 3 p$ orbitals for $\mathrm{Fe}$ and $1 s$ orbitals for $\mathrm{C}$ and $\mathrm{N}$ at the CCSD level (denoted as CCSD-rw), in conjunction with the weighted core-valence basis set aug-cc-pwCVTZ (Peterson \& Dunning 2002; Balabanov \& Peterson 2005). The inclusion of $1 s 2 s 2 p$ orbitals for $\mathrm{Fe}$ in the calculations is shown to have a negligible effect in the analogous $\mathrm{FeCN} / \mathrm{FeNC}$ systems (DeYonker 2015).

We also checked the possible role of relativistic effects through Douglas-Kroll-type calculations (Hess 1985, 1986; Jansen \& Hess 1989) at the CCSD level in conjunction with the basis set aug-cc-pVTZ-DK (denoted as CCSD-DK/aug-ccpVTZ-DK).

We estimated geometrical parameters, including additive corrections to those calculated at the CCSD(T)/aug-cc-pVQZ level through a composite procedure (Heckert et al. 2005, 2006; Huang \& Lee 2008). The composite bond distances are calculated by the following expression:

$$
r(\text { composite })=r(\mathrm{CBS})+\Delta r(\mathrm{CV})+\Delta r(\mathrm{DK}),
$$

where $r(\mathrm{CBS})$ corresponds to the complete basis set-limit evaluated through the $n^{-3}$ extrapolation formula (Helgaker et al. 1997) applied to the case $n=3,4$ (T, Q), employing the $\operatorname{CCSD}(\mathrm{T}) /$ aug-cc-pVTZ and $\operatorname{CCSD}(\mathrm{T}) /$ aug-cc-pVQZ optimized parameters. The corrections $\Delta r(\mathrm{CV})$ and $\Delta r$ (DK) account for the core-valence electron correlation and relativistic effects, and they are evaluated as:

$$
\begin{aligned}
\Delta r(\mathrm{CV})= & r(\mathrm{CCSD}-\mathrm{rw} / \text { aug-cc-pwCVTZ }) \\
& -r(\mathrm{CCSD} / \text { aug-cc-pVTZ }) \\
\Delta r(\mathrm{DK})= & r(\mathrm{CCSD}-\mathrm{DK} / \text { aug-cc-pVTZ-DK }) \\
& -r(\mathrm{CCSD} / \text { aug-cc-pVTZ }) .
\end{aligned}
$$

In a similar way, composite energies are obtained from the complete basis set-limit extrapolated energy, and corrected by the core-valence electron correlation, relativistic effects, and ZPV energy (calculated at the CCSD/aug-cc-pVTZ level):

$$
E(\text { composite })=E(\mathrm{CBS})+\Delta E(\mathrm{CV})+\Delta E(\mathrm{DK})+\mathrm{ZPVE} .
$$

Calculations were carried out with the Gaussian 09 program package (Frisch et al. 2009) and MOLPRO (Werner \& Knowles 2002).

\section{RESULTS AND DISCUSSION}

\section{1. $[\mathrm{C}, \mathrm{Fe}, \mathrm{H}, \mathrm{N}] \mathrm{System}$}

There are no previous studies on the $[\mathrm{C}, \mathrm{Fe}, \mathrm{H}, \mathrm{N}]$ system, therefore we have begun the study with a general search through the different isomers on the triplet and quintet potential energy surfaces. Other multiplicities were found to be not competitive. Different open-chain (linear and bent) 

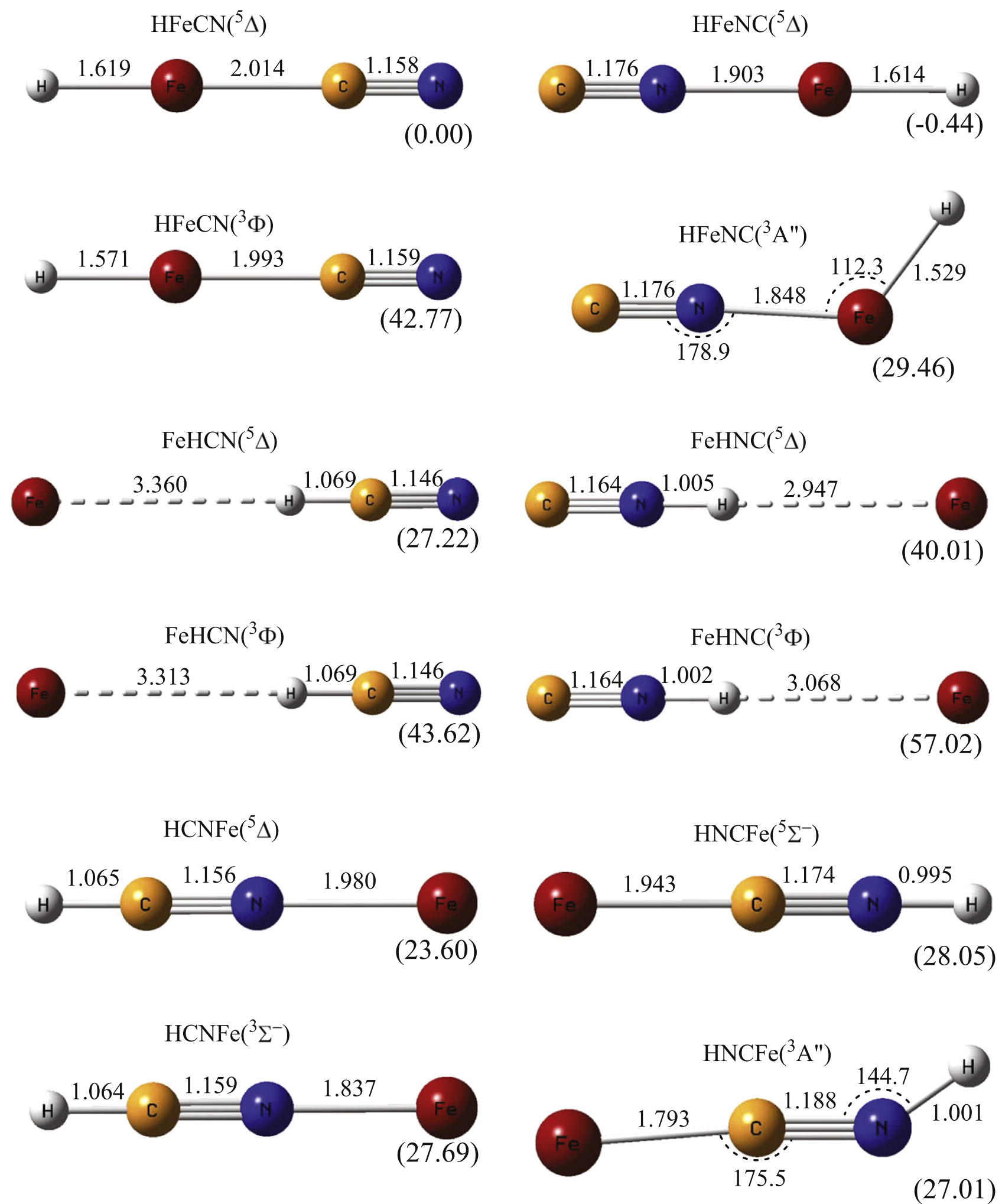

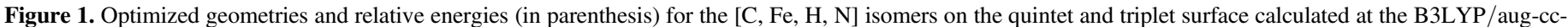

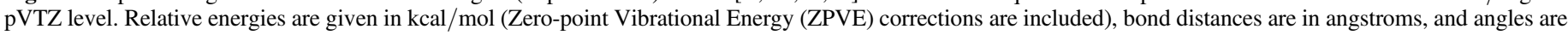
in degrees.

rearrangements are located with the iron atom located at different positions. It should be pointed out that we have not found stable cyclic structures where the iron atom is bonded to the carbon and nitrogen atoms. In Figure 1 we collect the structure and the relative energy for the $[\mathrm{C}, \mathrm{Fe}, \mathrm{H}, \mathrm{N}]$ isomers located on the triplet and quintet potential energy surfaces at the B3LYP/aug-cc-pVTZ level. Structures involving the cleavage of $\mathrm{CN}$ bonds were not included in Figure 1, because they are not competitive (located higher than $100 \mathrm{kcal} / \mathrm{mol}$ in energy). 
The most stable structures (Figure 1) correspond to the hydrides of iron cyanide/isocyanide, $\mathrm{HFeCN} / \mathrm{HFeNC}$ in their ${ }^{5} \Delta$ states. Both isomers have similar stability; their energy difference is less than $1 \mathrm{kcal} / \mathrm{mol}$ at the B3LYP/aug-cc-pVTZ level, and high-level calculations should be necessary to establish the most stable structure of this system. The lowestlying triplet states of $\mathrm{HFeCN}\left({ }^{3} \Phi\right)$ and $\mathrm{HFeNC}\left({ }^{3} \mathrm{~A}^{\prime \prime}\right)$ are located 42.77 and $29.90 \mathrm{kcal} / \mathrm{mol}$ above the corresponding quintet states, respectively. The isomers FeHCN ((hydrogen cyanide- $\kappa H)$ iron) and FeHNC ((hydrogen isocyanide- $\kappa H$ ) iron) correspond to the interaction between iron and either hydrogen cyanide or hydrogen isocyanide at the hydrogen atom. The ground state for both isomers is ${ }^{5} \Delta$, and they are located at 27.22 and $40.01 \mathrm{kcal} / \mathrm{mol}$, respectively, higher in energy than $\mathrm{HFeCN}\left({ }^{5} \Delta\right)$. The energetic gaps between FeHCN and FeHNC $(12.79 \mathrm{kcal} / \mathrm{mol})$ are similar to the energy difference between $\mathrm{HCN}$ and $\mathrm{HNC}(13.52 \mathrm{kcal} / \mathrm{mol}$ at the B3LYP/aug-cc-pVTZ level of theory), indicating that these isomers are intermolecular complexes between $\mathrm{Fe}$ and $\mathrm{HCN} /$ $\mathrm{HNC}$ molecules. This fact is also evident in the $\mathrm{H}-\mathrm{Fe}$ distances, which are close to $3.0 \AA$. In a similar way, the interaction between the iron atom and either $\mathrm{HCN}$ or $\mathrm{HNC}$ at either the nitrogen or carbon ends gives the isomers HCNFe (hydrogen cyanide- $\kappa N$ )iron, and HNCFe (hydrogen isocyanide- $\kappa C$ )iron. The electronic ground state is ${ }^{5} \Delta$ for isomer $\mathrm{HCNFe}$, whereas for $\mathrm{HNCFe}$ the ${ }^{3} \mathrm{~A}^{\prime \prime}$ state is $1.04 \mathrm{kcal} / \mathrm{mol}$ below the lowestlying quintet, ${ }^{5} \Sigma^{-}$, at the B3LYP/aug-cc-pVTZ level. These two isomers are located at 23.60 and $27.01 \mathrm{kcal} / \mathrm{mol}$, respectively, higher in energy than $\mathrm{HFeCN}$. It should be pointed out that these isomers cannot be considered intermolecular complexes because in them a bond between iron and either a nitrogen or a carbon atom is formed (see $\mathrm{Fe}-\mathrm{C} / \mathrm{N}$ bond distances in Figure 1).

We will focus on the lowest-lying state of each isomer, with $\mathrm{HNCFe}$ as the only exception, for which, as the energy difference calculated at the B3LYP level between triplet and quintet states, is only $1.04 \mathrm{kcal} / \mathrm{mol}$. We have considered both states. Geometries are optimized at the MP2/aug-cc-pVTZ and CCSD/aug-cc-pVTZ levels and electronic energies were refined through single-point calculations at the $\operatorname{CCSD}(\mathrm{T}) /$ aug-cc-pVQZ level over the CCSD/aug-cc-pVTZ geometries, namely the CCSD(T)/aug-cc-pVQZ//CCSD/aug-cc-pVTZ level. Table 1 reports optimized geometries and the corresponding energy differences are shown in Table 2. In this table we also include the calculated T1 diagnostic, and the $S^{2}$ value of the UHF/aug-cc-pVTZ wavefunction.

$\mathrm{T} 1$ diagnostic and spin contamination values can be used to determine the reliability of single-reference based results. A value of for the $\mathrm{T} 1$ diagnostic of less than 0.05 is proposed by Jiang et al. (2012) for obtaining reliable energetics and spectroscopic properties in $3 \mathrm{~d}$ transition metal compounds using single-reference based methods. From the results shown in Table 2, with the isomer HNCFe as the only exception (located more than $40 \mathrm{kcal} / \mathrm{mol}$ above the most stable isomer), it can be inferred that single-reference methods are suitable for describing these isomers (spin contamination values are negligible and $\mathrm{T} 1$ diagnostics are less than 0.05).

The optimized geometries calculated at the three levels of theory are similar, with the only exception being the isomer obtained by the interaction of the iron atom with the nitrogen of the HCN molecule. Table 1 features the predicted $\mathrm{Fe}-\mathrm{N}$ bond distance for the HCNFe $\left({ }^{5} \Delta\right)$ isomer $(3.5967$ and $3.8802 \AA$ at
Table 1

Optimized Geometries of the Different [H, C, N, Fe] Isomers at Different Levels of Theory in Conjunction with the aug-cc-pVTZ Basis Set

\begin{tabular}{|c|c|c|c|c|}
\hline \multirow{2}{*}{ Isomer } & & \multicolumn{3}{|c|}{ Level } \\
\hline & & B3LYP & MP2 & CCSD \\
\hline \multirow[t]{3}{*}{$\mathrm{HFeCN}\left({ }^{5} \Delta\right)$} & $\mathrm{d}(\mathrm{H}-\mathrm{Fe})$ & 1.6192 & 1.6349 & 1.6412 \\
\hline & $\mathrm{d}(\mathrm{Fe}-\mathrm{C})$ & 2.0138 & 2.0482 & 2.0343 \\
\hline & $\mathrm{d}(\mathrm{C}-\mathrm{N})$ & 1.1580 & 1.1554 & 1.1624 \\
\hline \multirow[t]{3}{*}{$\mathrm{HFeNC}\left({ }^{5} \Delta\right)$} & $\mathrm{d}(\mathrm{H}-\mathrm{Fe})$ & 1.6140 & 1.6314 & 1.6373 \\
\hline & $\mathrm{d}(\mathrm{Fe}-\mathrm{N})$ & 1.9031 & 1.9211 & 1.9214 \\
\hline & $\mathrm{d}(\mathrm{N}-\mathrm{C})$ & 1.1763 & 1.1867 & 1.1782 \\
\hline \multirow[t]{3}{*}{$\mathrm{FeHCN}\left({ }^{5} \Delta\right)$} & $\mathrm{d}(\mathrm{Fe}-\mathrm{H})$ & 3.3604 & 3.1484 & 3.4263 \\
\hline & $\mathrm{d}(\mathrm{H}-\mathrm{C})$ & 1.0689 & 1.0674 & 1.0665 \\
\hline & $\mathrm{d}(\mathrm{C}-\mathrm{N})$ & 1.1463 & 1.1666 & 1.1528 \\
\hline \multirow[t]{3}{*}{ FeHNC $\left({ }^{5} \Delta\right)$} & $\mathrm{d}(\mathrm{Fe}-\mathrm{H})$ & 2.9472 & 2.7329 & 3.0694 \\
\hline & $\mathrm{d}(\mathrm{H}-\mathrm{N})$ & 1.0052 & 1.0066 & 0.9986 \\
\hline & $\mathrm{d}(\mathrm{N}-\mathrm{C})$ & 1.1642 & 1.1771 & 1.1685 \\
\hline \multirow[t]{5}{*}{$\mathrm{HCNFe}\left({ }^{5} \mathrm{~A},{ }^{5} \Delta\right)$} & $\mathrm{d}(\mathrm{Fe}-\mathrm{N})$ & 1.9799 & 3.5967 & 3.8802 \\
\hline & $\mathrm{d}(\mathrm{N}-\mathrm{C})$ & 1.1563 & 1.1673 & 1.1524 \\
\hline & $\mathrm{d}(\mathrm{C}-\mathrm{H})$ & 1.0650 & 1.0651 & 1.0657 \\
\hline & $<\mathrm{Fe}-\mathrm{N}-\mathrm{C}$ & 180. & 144.4 & 180. \\
\hline & $<$ dih & & 182.0 & \\
\hline \multirow[t]{5}{*}{$\operatorname{HNCFe}\left({ }^{5} \Sigma^{-},{ }^{5} \mathrm{~A}^{\prime}\right)$} & $\mathrm{d}(\mathrm{Fe}-\mathrm{C})$ & 1.9429 & 1.9758 & 1.9529 \\
\hline & $\mathrm{d}(\mathrm{C}-\mathrm{N})$ & 1.1739 & 1.2062 & 1.2117 \\
\hline & $\mathrm{d}(\mathrm{N}-\mathrm{H})$ & 0.995 & 1.0188 & 1.0161 \\
\hline & $<\mathrm{Fe}-\mathrm{C}-\mathrm{N}$ & 180. & 173.4 & 173.1 \\
\hline & $<\mathrm{C}-\mathrm{N}-\mathrm{H}$ & 180. & 127.0 & 124.2 \\
\hline \multirow[t]{5}{*}{$\mathrm{HNCFe}\left({ }^{3} \mathrm{~A}^{\prime \prime}\right)$} & $\mathrm{d}(\mathrm{Fe}-\mathrm{C})$ & 1.7926 & 1.8783 & 1.8146 \\
\hline & $\mathrm{d}(\mathrm{C}-\mathrm{N})$ & 1.1884 & 1.2126 & 1.2104 \\
\hline & $\mathrm{d}(\mathrm{N}-\mathrm{H})$ & 1.0014 & 1.0169 & 1.0116 \\
\hline & $<\mathrm{Fe}-\mathrm{C}-\mathrm{N}$ & 175.5 & 173.9 & 175.5 \\
\hline & $<\mathrm{C}-\mathrm{N}-\mathrm{H}$ & 144.7 & 126.9 & 126.0 \\
\hline
\end{tabular}

Note. Bond distances are given in angstroms and angles are in degrees.

the MP2 and CCSD levels, respectively), highlighting the fact that at these levels it is best described as an intermolecular complex. We found that this complex is $10.36 \mathrm{kcal} / \mathrm{mol}$ more stable than the molecular structure located at the B3LYP level, at the $\operatorname{CCSD}(\mathrm{T}) /$ aug-cc-pVQZ//CCSD/aug-cc-pVTZ level. All our attempts to locate this complex at the B3LYP level failed. On the other hand, the isomer comes from the interaction of an iron atom with a carbon atom in the HNC molecule, HNCFe, and no intermolecular complex has been characterized in either in its triplet or its quintet state.

A comparison between the geometrical parameters of the hydrides of iron cyanide/isocyanide and those of the molecules $\mathrm{HFe}$ and $\mathrm{CN}(\mathrm{H}-\mathrm{Fe}$ and $\mathrm{C}-\mathrm{N}$ distances are 1.5992 and $1.1672 \AA$ at the CCSD/aug-cc-pVTZ level, respectively) shows that when both isomers are formed, the $\mathrm{H}-\mathrm{Fe}$ bond distance increases with respect to that of HFe alone. However, we note that when an iron atom is bonded through the carbon atom the $\mathrm{C}-\mathrm{N}$ bond distance slightly decreases, whereas it slightly increases if the bonding occurs through the nitrogen atom. We have found a similar behavior in our previous study on hydrides of zinc cyanide/isocyanide (Redondo et al. 2015). On the other hand, isomers whose structures are described as intermolecular complexes present distances for $\mathrm{H}-\mathrm{C} / \mathrm{N}$ and $\mathrm{C}-\mathrm{N}$ that are almost identical to those corresponding to hydrogen cyanide (H-C and C-N are 1.065 and $1.1526 \AA$ at the CCSD/aug-cc-pVTZ level, respectively) or hydrogen isocyanide $(\mathrm{H}-\mathrm{N}$ and $\mathrm{C}-\mathrm{N}$ are 0.9951 and $1.1686 \AA$ at the CCSD/ aug-cc-pVTZ level, respectively). 


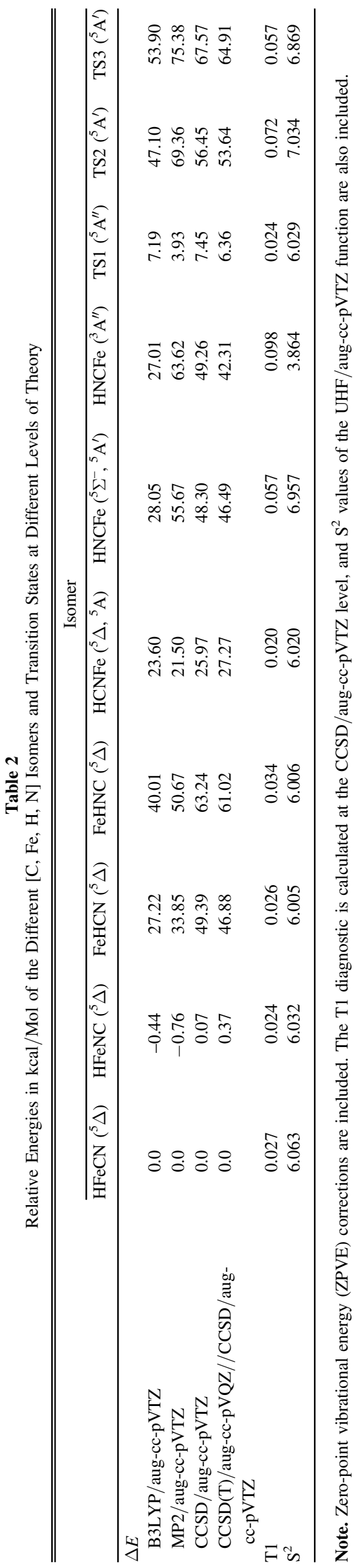


Table 3

Dissociation Energies, in $\mathrm{kcal} / \mathrm{mol}$, Calculated at the CCSD(T)/aug-ccpVQZ//CCSD/aug-cc-pVTZ level, ZPVE Included

\begin{tabular}{lr}
\hline \hline Process & DE \\
\hline $\mathrm{HFeCN} \rightarrow \mathrm{HFe}+\mathrm{CN}$ & 113.7 \\
$\mathrm{HFeNC} \rightarrow \mathrm{HFe}+\mathrm{CN}$ & 113.3 \\
$\mathrm{FeHCN} \rightarrow \mathrm{Fe}+\mathrm{HCN}$ & 0.4 \\
$\mathrm{FeHNC} \rightarrow \mathrm{Fe}+\mathrm{HNC}$ & 0.7 \\
$\mathrm{HCNFe} \rightarrow \mathrm{Fe}+\mathrm{HCN}$ & 20.0 \\
$\mathrm{HNCFe} \rightarrow \mathrm{Fe}+\mathrm{HNC}$ & 19.5 \\
\hline
\end{tabular}

It is readily seen in Table 2, that the lowest-lying structures are $\mathrm{HFeCN}$ and $\mathrm{HFeNC}$, and the energy difference between them is less than $1 \mathrm{kcal} / \mathrm{mol}$, regardless of the level of calculation employed. It seems that improving the level of calculation and the size of the basis set favors the isomer $\mathrm{HFeCN}$, which is located $0.37 \mathrm{kcal} / \mathrm{mol}$ lower in energy than $\mathrm{HFeNC}$ at the CCSD(T)/aug-cc-pVQZ//CCSD/aug-cc-pVTZ level. All levels of theory employed predict that the structures arising from the interaction of the $\mathrm{Fe}$ atom with $\mathrm{HCN} / \mathrm{HNC}$ molecules are clearly located above the hydride type isomers (energy differences with respect to $\mathrm{HFeCN}$ are higher than $20 \mathrm{kcal} / \mathrm{mol}$ ). Among them, the interaction of an iron atom with $\mathrm{C} / \mathrm{N}$ atoms is more favorable than interaction through a hydrogen atom, and the isomers derived from $\mathrm{HCN}$ are more stable than the corresponding isomers from HNC.

In addition, the stability of the different isomers is analyzed in terms of the dissociation energies. The following processes are considered: $\mathrm{HFe}(\mathrm{CN}) \rightarrow \mathrm{HFe}+\mathrm{CN}, \quad \mathrm{FeH}(\mathrm{CN}) \rightarrow$ $\mathrm{Fe}+\mathrm{H}(\mathrm{CN})$, and $\mathrm{H}(\mathrm{CN}) \mathrm{Fe} \rightarrow \mathrm{Fe}+\mathrm{H}(\mathrm{CN})$. The results obtained at the $\operatorname{CCSD}(\mathrm{T}) /$ aug-cc-pVQZ//CCSD/aug-ccpVTZ level, including ZPE corrections, are shown in Table 3.

We can see that hydrides of iron cyanide/isocyanide have high dissociation energies (about $113 \mathrm{kcal} / \mathrm{mol}$ at the CCSD (T)/aug-cc-pVTZ//CCSD/aug-cc-pVTZ level), therefore they are stable against fragmentation into $\mathrm{HFe}$ and $\mathrm{CN}$. Isomers arising from the interaction of $\mathrm{Fe}$ with $\mathrm{HCN} / \mathrm{HNC}$ are less stable in relation to dissociation. Particularly, when the interaction of an iron atom is through a hydrogen atom, the dissociation energy is less than $1 \mathrm{kcal} / \mathrm{mol}$. These values are similar to those found in the corresponding structures with magnesium and zinc. However, the interaction between the iron atoms, and either $\mathrm{HCN}$ or $\mathrm{HNC}$ at either the nitrogen or carbon ends, is more favorable for iron atoms than for magnesium and zinc (Gronowski \& Kołos 2013; Redondo et al. 2015).

Finally, we will analyze the isomerization processes between the isomers localized for the $[\mathrm{C}, \mathrm{Fe}, \mathrm{H}, \mathrm{N}]$ system shown in Figure 2. Relative energies of the transition states at different levels of theory are also included in Table 2. First, we take into account the interconversion between the two most stable isomers, HFeCN and HFeNC. Transition state, denoted as TS1, connects both structures, and is located $5.99 \mathrm{kcal} / \mathrm{mol}$ (approximately $720 \mathrm{~K}$ ) above the HFeNC isomer. This barrier suggests that the interconversion process between the two most stable isomers should have a very slow rate. Therefore, even though cosmological timescales are extremely long, it is likely that both isomers could be present simultaneously if efficient synthesis processes giving both are viable.

The van der Waals complexes, FeHCN and FeHNC, could be formed by direct interaction between an iron atom and $\mathrm{HCN}$ or HNC. We have considered the isomerization processes between these complexes and the corresponding lowest-lying isomers, $\mathrm{HFeCN}$ and $\mathrm{HFeNC}$. The transition state connecting FeHCN with $\mathrm{HFeCN}$, TS2, is located $6.76 \mathrm{kcal} / \mathrm{mol}(810 \mathrm{~K})$ above the FeHCN isomer, and the isomerization of FeHNC into HFeNC involves transition state TS3, which is placed $3.89 \mathrm{kcal} / \mathrm{mol}(470 \mathrm{~K})$ above the FeHNC isomer. Both processes have a net activation barrier, indicating that the most stable isomers of the $[\mathrm{C}, \mathrm{Fe}, \mathrm{H}, \mathrm{N}]$ system, $\mathrm{HFeCN}$ and $\mathrm{HFeNC}$, cannot efficiently originate from FeHCN and FeHNC, respectively, in space conditions. It seems that one synthetic route of these compounds could be, as suggested by Cabezas et al. (2013), is the case of $\mathrm{HMgNC}$, through the radiative association of $\mathrm{Fe}^{+}$with cyanopolyynes $\left(\mathrm{HC}_{2 \mathrm{n}+1} \mathrm{H}\right)$, followed by the dissociative recombination of the cation complex. A similar route is also proposed for the formation of $\mathrm{FeCN}$ (Zack et al. 2011); this suggests a common origin and therefore $\mathrm{HFeCN} / \mathrm{HFeNC}$ and $\mathrm{FeCN}$ could be present in similar sources.

The previous results show that both $\mathrm{HFeCN}$ and $\mathrm{HFeNC}$ isomers are expected to be the most reasonable targets detected in the interstellar medium and characterized in the laboratory. We will focus on the molecular structure of these isomers in the next section.

\subsection{HFeCN and HFeNC Isomers}

The results of the improved geometrical parameters and rotational constants for hydrides of iron cyanide/isocyanide computed at different theory levels are given in Table 4 . The computed rotational constants correspond to equilibrium structures. In order to check the readability of our results, based on single-reference calculations, we have carried out CASSCF/aug-cc-pVTZ calculations on the two isomers. The weight $\mathrm{C}_{\mathrm{o}}^{2}$ of the ${ }^{5} \Delta$ configuration in the CASSCF wavefunction is higher than 0.9 (0.912 and 0.925 for $\mathrm{HFeCN}$ and $\mathrm{HFeNC}$, respectively). These values, in addition to the previous comment on S2 and T1 diagnostics, show that these isomers are properly described by one-reference, wavefunction-based methods.

From Table 4 we can see that the effect of the inclusion of triple excitations on geometrical parameters at the CCSD(T) level is a lowering of $\mathrm{H}-\mathrm{Fe}$ and $\mathrm{Fe}-\mathrm{C} / \mathrm{N}$ bond distances and an increase of the $\mathrm{CN}$ one. When we increase the size of the basis set, going from aug-cc-pVTZ to aug-cc-pVQZ, the H-Fe bond distance lengthens, whereas the $\mathrm{Fe}-\mathrm{C} / \mathrm{N}$ and $\mathrm{CN}$ bonds shorten. The inclusion of core-valence correlation by including $3 s 3 p$ orbitals for $\mathrm{Fe}$ and $1 s$ orbitals for $\mathrm{C}$ and $\mathrm{N}$ at the CCSD-rw level results in a shortening of all bond lengths. Similar effects on bond distances are found by DeYonker (2015) for similar $\mathrm{FeCN} / \mathrm{FeNC}$ systems. The inclusion of relativistic effects in optimized geometries decreases the $\mathrm{H}-\mathrm{Fe}$ and $\mathrm{Fe}-\mathrm{C} / \mathrm{N}$ bond distances. Our predicted "composite" geometries and rotational constants take into account these effects. The computed dipolar moments, also included in Table 4, are similar for both isomers (about $3.00 \mathrm{D}$ ), and these relatively high values make these compounds capable of being affordably detected by rotational spectroscopy.

Relative energies between $\mathrm{HFeCN}$ and $\mathrm{HFeNC}$ using different additive corrections to the couplet cluster energy in the composite method are reported in Figure 3. As we have previously seen from the results shown in Table 2, both isomers present a similar stability; their energy difference is less than $1 \mathrm{kcal} / \mathrm{mol}$. In a previous work on $\mathrm{FeCN} / \mathrm{FeNC}$, DeYonker 


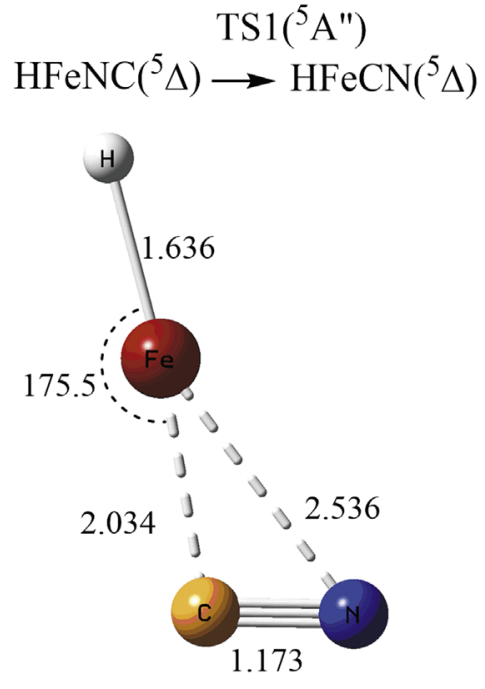

(5.99)

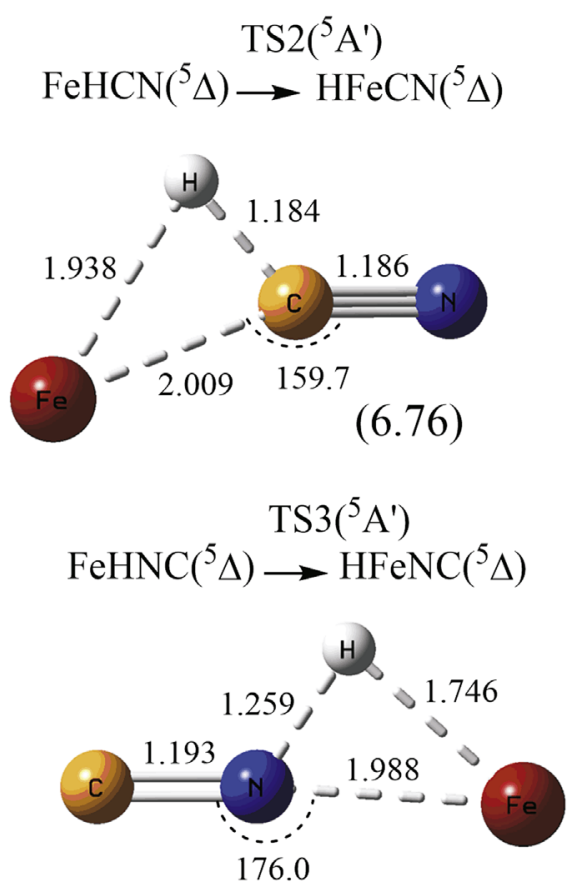

(3.89)

Figure 2. Optimized geometries at the CCSD/aug-cc-PVTZ level for the transition states corresponding to different isomerization processes between [C, Fe, H, N] isomers. Bond distances are given in angstroms and angles are in degrees. Isomerization barriers with respect to the less stable isomer (in parenthesis) calculated at the $\mathrm{CCSD}(\mathrm{T}) /$ aug-cc-pVQZ//CCSD/aug-cc-PVTZ level are given in $\mathrm{kcal} / \mathrm{mol}$.

Table 4

Optimized Geometries, Equilibrium Rotational Constants (MHz), and Dipole Moment (Debye) for HFeCN and HFNC Isomers Obtained at Different Levels of Theory

\begin{tabular}{|c|c|c|c|c|c|c|c|c|c|c|}
\hline \multirow{2}{*}{ Level } & \multicolumn{5}{|c|}{$\mathrm{HFeCN}$} & \multicolumn{5}{|c|}{$\mathrm{HFeNC}$} \\
\hline & $\mathrm{d}(\mathrm{H}-\mathrm{Fe})$ & $\mathrm{d}(\mathrm{Fe}-\mathrm{C})$ & $\mathrm{d}(\mathrm{C}-\mathrm{N})$ & $B_{\mathrm{e}}$ & $\mu$ & $\mathrm{d}(\mathrm{H}-\mathrm{Fe})$ & $\mathrm{d}(\mathrm{Fe}-\mathrm{N})$ & $\mathrm{d}(\mathrm{C}-\mathrm{N})$ & $B_{\mathrm{e}}$ & $\mu$ \\
\hline CCSD/aug-cc-pVTZ & 1.6412 & 2.0343 & 1.1624 & 3596.84 & 3.56 & 1.6373 & 1.9214 & 1.1782 & 4119.95 & 3.32 \\
\hline CCSD(T)/aug-cc-pVTZ & 1.6388 & 2.0241 & 1.1690 & 3611.09 & $\ldots$ & 1.6346 & 1.9173 & 1.1861 & 4117.43 & $\ldots$ \\
\hline CCSD(T)/aug-cc-pVQZ & 1.6427 & 2.0213 & 1.1656 & 3623.32 & $\cdots$ & 1.6381 & 1.9146 & 1.1828 & 4131.36 & $\cdots$ \\
\hline CCSD-rw/aug-cc-pwCVTZ & 1.6344 & 2.0283 & 1.1586 & 3619.13 & 3.57 & 1.6303 & 1.9162 & 1.1744 & 4143.79 & 3.56 \\
\hline CCSD-DK/aug-cc-pVTZ-DK & 1.6339 & 2.0191 & 1.1623 & 3635.64 & $\ldots$ & 1.6290 & 1.9121 & 1.1781 & 4149.41 & $\cdots$ \\
\hline Composite & 1.6315 & 1.9980 & 1.1591 & 3693.35 & $\cdots$ & 1.6253 & 1.8982 & 1.1765 & 4194.82 & $\cdots$ \\
\hline
\end{tabular}

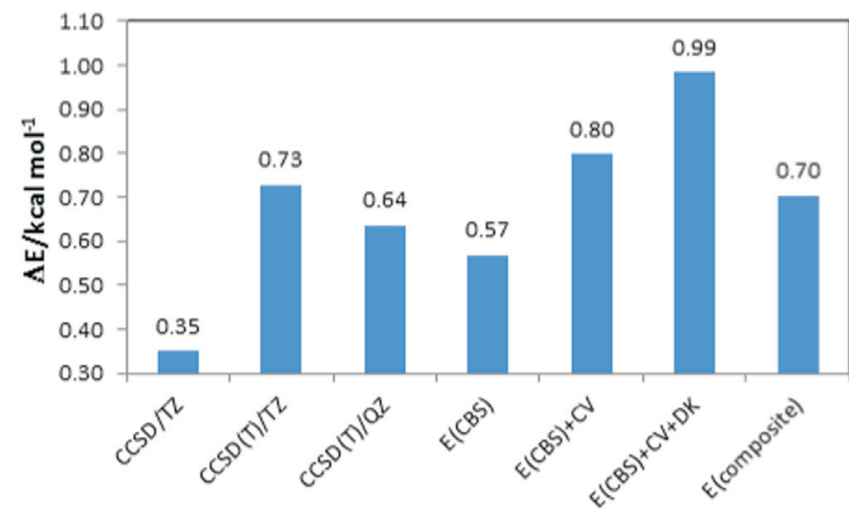

Figure 3. Relative energies, in $\mathrm{kcal} / \mathrm{mol}$, between $\mathrm{HFeCN}$ and $\mathrm{HFeNC}$ (with the $\mathrm{HFeCN}$ as reference), using different additive corrections to the coupled cluster energy in the composite method.

(2015) suggested that for correctly predicting the energy in these systems, the effects of core-valence electron correlation and CBS energy extrapolation must be added to singlereference couplet cluster calculation. We have calculated these corrections to evaluate the energy difference between $\mathrm{HFeCN}$ and $\mathrm{HFeNC}$ isomers. In Figure 3 we can see that the inclusion of triple excitations on energy at the CCSD(T)/aug-cc-pVTZ level stabilize the isomer $\mathrm{HFeCN}$ over $\mathrm{HFeNC}$. An opposite effect is found when the size of the basis set is increased; the isomer $\mathrm{HFeNC}$ is stabilized. The addition of core-valence correlation and relativistic effects to the extrapolated CBS energy favors the $\mathrm{HFeCN}$ isomer stability, whereas ZPVE corrections have the opposite effect. We have also computed the spin-orbit corrections (SOCs) for both isomers. The obtained values are -402.79 and $-402.43 \mathrm{~cm}^{-1}$ for $\mathrm{HFeCN}$ and $\mathrm{HFeNC}$, respectively. Therefore, SOsC do not change the relative energy between both isomers. All the levels employed predict that the isomer $\mathrm{HFeCN}$ is slightly more stable than the $\mathrm{HFeNC}$ isomer. Our computed composite value, which corrects the $\operatorname{CCSD}(\mathrm{T})$ energy with CBS energy extrapolation, corevalence energy correlation, relativistic effects, and ZPVE corrections, predicts that $\mathrm{HFeCN}$ is $0.70 \mathrm{kcal} / \mathrm{mol}$ more stable than HFeNC. This result shows that both isomers are quasiisoenergetic and from a thermodynamic point of view both are possible targets for experimental search. The relative 
Table 5

Vibrational Frequencies $\left(\mathrm{cm}^{-1}\right)$ and IR Intensities $(\mathrm{km} / \mathrm{mol})$ of the $\mathrm{HFeCN}$ and $\mathrm{HFeNC}$ Isomers

\begin{tabular}{|c|c|c|c|c|c|c|c|c|c|c|}
\hline & \multicolumn{5}{|c|}{$\mathrm{HFeCN}$} & \multicolumn{5}{|c|}{$\mathrm{HFeNC}$} \\
\hline & \multicolumn{2}{|c|}{$\begin{array}{l}\text { CCSD/aug- } \\
\text { cc-pVTZ }\end{array}$} & \multicolumn{2}{|c|}{$\begin{array}{l}\text { CCSD-rw/ } \\
\text { aug-cc- } \\
\text { pwCVTZ }\end{array}$} & \multirow{2}{*}{$\begin{array}{c}\text { CCSD-DK/aug-cc-pVTZ-DK } \\
\omega\end{array}$} & \multicolumn{2}{|c|}{$\begin{array}{l}\text { CCSD/aug- } \\
\text { cc-pVTZ }\end{array}$} & \multicolumn{2}{|c|}{$\begin{array}{c}\text { CCSD-rw/ } \\
\text { aug-cc- } \\
\text { pwCVTZ }\end{array}$} & \multirow{2}{*}{$\begin{array}{c}\text { CCSD-DK/aug-cc-pVTZ-DH } \\
\omega \\
\end{array}$} \\
\hline & $\omega$ & $I$ & $\omega$ & $I$ & & $\omega$ & $I$ & $\omega$ & $I$ & \\
\hline$\alpha \mathrm{CN}$ stretching & 2264 & 32.1 & 2277 & 32.5 & 2264 & 2136 & 307.7 & 2147 & 312.8 & 2135 \\
\hline$\alpha \mathrm{FeH}$ stretching & 1797 & 258.9 & 1811 & 269.1 & 1814 & 1803 & 242.5 & 1818 & 251.7 & 1820 \\
\hline$\pi \mathrm{FeCN}(\mathrm{NC})$ bending & 185 & 27.7 & 188 & 32.6 & 186 & 117 & 5.1 & 122 & 5.7 & 116 \\
\hline
\end{tabular}

abundance of $\mathrm{HFeCN} / \mathrm{HFeNC}$ isomers will depend on kinetic factors.

The calculated harmonic vibrational frequencies and infrared (IR) intensities for both isomers, $\mathrm{HFeCN}$ and $\mathrm{HFeNC}$, are given in Table 5. This information could be useful for an eventual detection in the gas phase of hydride or iron cyanide/ isocyanide through IR spectroscopy. The inclusion of the corevalence energy correlation in the calculation, the CCSD-rw level, causes a slight increase in the vibrational frequencies that is related to the effect found on the optimized geometries. As we have previously mentioned, CCSD-rw distances are shorter than CCSD distances. Vibrational frequencies calculated at the CCSD-DK level are relatively close to those computed at the CCSD level. Relativistic effects in the computations of vibrational frequencies do not have a significant contribution for this system. The predicted IR spectra are similar for both isomers; the only difference is the IR intensities corresponding to the $\mathrm{C}-\mathrm{N}$ stretching frequency that is the most intense for the isomer $\mathrm{HFeNC}(311.6 \mathrm{~km} / \mathrm{mol})$, whereas for $\mathrm{HFeCN}$ isomer its intensity is relatively small $(32.5 \mathrm{~km} / \mathrm{mol})$.

\section{CONCLUSIONS}

We have carried out a theoretical study of [C, H, Fe, N] isomers on the quintet and triplet potential energy surfaces by means of different quantum chemical approaches. The most stable structures correspond to the hydrides of iron cyanide/ isocyanide, $\mathrm{HFeCN} / \mathrm{HFeNC}$, (hydrogen cyanide/isocyanide$\kappa H$ ) iron, FeHCN/FeHNC, and (hydrogen cyanide/isocyanide- $\kappa N / C$ ) iron, $\mathrm{HCNFe} / \mathrm{HNCFe}$. The lowest-lying state for these isomers is a quintet, with the only exception being $\mathrm{HNCFe}$, where the ${ }^{3} \mathrm{~A}^{\prime \prime}$ is located $4.18 \mathrm{kcal} / \mathrm{mol}$ below ${ }^{5} \mathrm{~A}^{\prime}$ at the CCSD(T)/aug-cc-pVQZ//CCSD/aug-cc-pVTZ level.

The lowest-lying structures are $\mathrm{HFeCN}$ and $\mathrm{HFeNC}$ in their ${ }^{5} \Delta$ states, which are located more than $20 \mathrm{kcal} / \mathrm{mol}$ lower in energy than the other isomers, and the energy difference between them is less than $1 \mathrm{kcal} / \mathrm{mol}$, regardless of the level of calculation employed. Among the isomers arising from the interaction of iron with $\mathrm{HCN} / \mathrm{HNC}$, we have found that the interaction of iron atoms with $\mathrm{C} / \mathrm{N}$ atoms is more favorable than interaction through hydrogen atoms, and the isomers derived from $\mathrm{HCN}$ are more stable than the corresponding $\mathrm{HNC}$ isomers.

The intermolecular isomers FeHCN and FeHNC could be formed by direct interaction between an iron atom and $\mathrm{HCN}$ or HNC. The isomerization processes between $\mathrm{FeHCN}$ and FeHNC, which could be formed by the direct interaction between an iron atom and $\mathrm{HCN}$ or $\mathrm{HNC}$, and the corresponding lowest-lying isomers $\mathrm{HFeCN}$ and $\mathrm{HFeNC}$, involve net activation barriers $(6.76 \mathrm{kcal} / \mathrm{mol}(810 \mathrm{~K})$ and $3.89 \mathrm{kcal} / \mathrm{mol}$
(470 K) at the CCSD(T)/aug-cc-pVQZ//CCSD/aug-cc-pVTZ level, respectively), indicating that the $\mathrm{HFeCN}$ and $\mathrm{HFeNC}$ isomers cannot efficiently originate from $\mathrm{FeHCN}$ and FeHNC, respectively, under space conditions. It seems that one synthetic route for these compounds could be through the radiative association of $\mathrm{Fe}^{+}$with cyanopolyynes $\left(\mathrm{HC}_{2 \mathrm{n}+1} \mathrm{H}\right)$, followed by the dissociative recombination of the cation complex.

The interconversion process between the $\mathrm{HFeCN}$ and $\mathrm{HFeNC}$ isomers has an extremely slow rate (a net activation barrier of about $6 \mathrm{kcal} / \mathrm{mol}$ ), therefore, under interstellar conditions, both isomers are expected to be reasonable targets for detection in the interstellar medium and for characterization in the laboratory. In order to give more reliable geometrical parameters for these isomers we have included corrections at the $\operatorname{CCSD}(\mathrm{T})$ level for a complete basis set, core-valence correlation energy, relativistic effects, and composite calculations. At this level, both isomers are quasi-isoenergetics ( $\mathrm{HFeCN}$ is located $0.70 \mathrm{kcal} / \mathrm{mol}$ below $\mathrm{HFeNC}$ at the composite level), and from a thermodynamic point of view they could be possible targets for an experimental search. The relative abundance of $\mathrm{HFeCN} / \mathrm{HFeNC}$ isomers will depend on kinetic factors. In addition, predicted IR spectra for hydride or iron cyanide/isocyanide are reported that could help them be detected in the gas phase through IR spectroscopy.

Financial support from the "Junta de Castilla y León" (GrantVA077U13) is gratefully acknowledged. This work results from a collaboration of the COST Action TD 1308.

\section{REFERENCES}

Balabanov, N. B., \& Peterson, K. A. 2005, JChPh, 123, 064107

Becke, A. D. 1988, JChPh, 88, 2547

Cabezas, C., Cernicharo, J., Alonso, J. L., et al. 2013, ApJ, 775, 133

Cernicharo, J., \& Guélin, M. 1987, A\&A, 183, L10

DeYonker, N. J. 2015, JPCA, 119, 215

DeYonker, N. J., Yamaguchi, Y., Allen, W. D., et al. 2004, JChPh, 120, 4726

Dunbar, R. C., \& Petrie, S. 2002, ApJ, 564, 792

Dunning, T. H. 1989, JChPh, 90, 1007

Flory, M. A., \& Ziurys, L. M. 2011, JChPh, 135, 184303

Frisch, M. J., Trucks, G. W., Schlegel, H. B., et al. 2009, Gaussian 09 (Wallingford CT: Gaussian, Inc.)

Gronowski, M., \& Kołos, R. 2013, JPCA, 117, 4455

Guélin, M., Lucas, R., \& Cernicharo, J. 1993, A\&A, 280, L19

Guélin, M., Muller, S., Cernicharo, J., et al. 2000, A\&A, 363, L9

Guélin, M., Muller, S., Cernicharo, J., et al. 2004, A\&A, 426, L49

Heckert, M., Kalay, M., \& Gauss, J. 2005, MPh, 103, 2109

Heckert, M., Kalay, M., Tew, D. P., et al. 2006, JChPh, 125, 044108

Helgaker, T., Klopper, W., Koch, H., \& Noga, J. 1997, JChPh, 106, 9639

Hernández Vera, M., Lique, F., Klos, J., et al. 2015, MNRAS, 451, 5717

Hess, B. A. 1985, PhRvA, 32, 756

Hess, B. A. 1986, PhRvA, 33, 3742 
Hirano, T., Amano, M., Mitsui, Y., et al. 2007, JMoSp, 243, 267

Hirano, T., Okuda, R., Nagashima, U., et al. 2006, JMoSp, 236, 234

Huang, T., \& Lee, T. J. 2008, JChPh, 128, 044312

Jansen, G., \& Hess, B. A. 1989, PhRvA, 39, 6016

Jiang, W., DeYonker, N. J., \& Wilson, A. K. 2012, J. Chem. Theory Comput., 8,460

Kamiński, T., Gottlieb, C. A., \& Menten, K. M. 2013, A\&A, 551, A113

Kawaguchi, K., Kagi, E., Hirano, T., et al. 1993, ApJL, 406, L39

Lee, C., Yang, W., \& Parr, R. G. 1988, PhRvB, 37, 785

Lee, T. J., \& Taylor, P. R. 1989, Int. J. Quant. Chem. Symp, S23, 199

Maier, G., Reisenauer, H. P., Egenolf, H., \& Glatthaar, J. 1998, European J. Org. Chem, 1998, 1307

Møller, C., \& Plesset, M. 1934, PhRv, 46, 618

Peterson, K. A., \& Dunning, T. H. 2002, JChPh, 117, 10548

Petrie, S. 1996, MNRAS, 282, 807

Pulliam, R. L., Savage, C., Agúndez, M., et al. 2010, ApJL, 725, L181

Raghavachari, K., Trucks, G. W., Pople, J. A., \& Head-Gordon, M. 1989, CPL, 157,479
Rayón, V. M., Redondo, P., Valdes, H., et al. 2007, JPCA, 111, 6334

Redondo, P., Largo, A., Vega-Vega, A., \& Barrientos, C. 2015, JChPh, 142, 184301

Sanz, M. E., McCarthy, M. C., \& Thaddeus, P. 2002, ApJL, 577, L71

Sun, M., Apponi, A. J., \& Ziurys, L. M. 2009, JChPh, 130, 034309

Tenenbaum, E. D., \& Ziurys, L. M. 2009, ApJL, 693, L59

Tenenbaum, E. D., \& Ziurys, L. M. 2010, ApJL, 712, L93

Tsuji, T. 1973, A\&A, 23, 411

Turner, B. E., Steimle, T. C., \& Meerts, L. 1994, ApJL, 426, L97

Walmsley, C. M., Bachiller, R., Pineau des Forêts, G., \& Schilke, P. 2002, ApJL, 566, L109

Werner, H.-J., \& Knowles, P. J. 1985a, JChPh, 82, 5053

Werner, H.-J., \& Knowles, P. J. 1985b, CPL, 115, 259

Woon, D. E., \& Dunning, T. H. 1993, JChPh, 98, 1358

Zack, L. N., Halfen, D. T., \& Ziurys, L. M. 2011, ApJL, 733, L36

Ziurys, L. M., Apponi, A. J., Guélin, M., \& Cernicharo, J. 1995, ApJL, 445, L47

Ziurys, L. M., Savage, C., Highberger, J. L., et al. 2002, ApJL, 564, L45 